\title{
Cytotoxic CD4+ cells in Chronic Lymphocytic Leukaemia: An extended immunophenotypic analysis examining their association with cytomegalovirus serostatus and similarities with cytotoxic CD8+ cells.
}

Maria A V Marzolini, Ayse Akarca, Karen Teng, Teresa Marafioti, Satyen H Gohil, Amit Nathwani, Evan Newell, Sergio A Quezada, Karl S Peggs

\section{Introduction}

CD4+ T lymphocytes play a fundamental role in the adaptive immune system and traditionally their principle function has been seen as one of a 'helper' cell, interacting with and activating fellow immune cells to orchestrate and regulate the degree of immune response. There is a subset of $\mathrm{CD} 4+\mathrm{T}$ lymphocytes, however, that have the ability to acquire a cytotoxic phenotype. These cytotoxic CD4+ cells have been shown to have anti-viral functions but also they have previously been reported to exhibit anti-tumour effects in murine models of solid organ malignancies e.g. melanoma.

The aim of this research was to identify, quantify and characterise cytotoxic CD4+ cells (CD4+ CTLs) in patients with Chronic Lymphocytic Leukaemia (CLL), to examine their association with cytomegalovirus (CMV) and to investigate the phenotype differences of the CD4+ CTLs isolated from patients who were CMV seropositive and seronegative.

\section{Methods}

The peripheral blood (PB) from 90 patients with stable CLL (that was not requiring therapy) was collected after ethical approval and consent had been obtained. Flow cytometry was performed to identify and quantify the level of cytotoxic CD4+ cells (as defined as CD4+ Granzyme B+ cells) and to characterise the extended immunophenotype of the cells including transcription factors (including GATA-3, FoxP3, Eomes and Tbet) and co-inhibitory and co-stimulatory molecules (including LAG-3, TIM3, CTLA-4, PD-1, ICOS, OX40 and 4-1BB).

In a subset of patients, serial samples of PB were collected to assess whether the quantity of cytotoxic CD4 cells changed over time in untreated CLL patients.

Mass cytometry was performed to detect a broad range of surface molecules and to compare the extended phenotype of CD4+ (including CD4+ CTLs) and CD8+ cells in patients who were CMV seropositive and CMV seronegative in 14 patients with untreated CLL.

An analysis of the CLL tumour environment was performed using multi-colour immunohistochemistry to detect CD4+ CTLs in paired bone marrow trephine and lymph node biopsies from a subset of patients. The expression of co-inhibitory and co-stimulatory molecules on the CD4+CTLs was also examined in the bone marrow and lymph node biopsies.

\section{Results}

The median age of the patients was 67 years (range: $41-90$ years) with 29 females and 61 males. Fourteen patients had previously required treatment for CLL but were not currently on treatment and 76 patients had never required treatment.

There was a significant increase in the percentage of CD4+ CTLs in the PB of those patients who were $C M V$ seropositive when compared to those who were $C M V$ seronegative $(P<0.0001)$. Forty-five 
patients had PB samples collected at two different time-points and the median time between the two samples was 189 days (range: 7-735 days). The median difference in CD4+CTL values between the 2 samples for this group of patients was $0.19 \%$ (range: $-25-46 \%$ ).

The extended immunophenotyping of the CD4+ CTLs showed that they had a phenotype more similar to cytotoxic CD8+ cells than non-cytotoxic CD4+ cells, with respect to both their transcription factor and checkpoint molecule expression.

The mass cytometry analysis showed that there were significant differences in the expression of surface markers on CD4+ CTLs present in the PB of patients who were CMV seropositive compared with those who were CMV seronegative.

The CD4+ CTLs were identified in both the bone marrow and lymph node biopsies. There was a significantly higher percentage of CD4+CTLs in the bone marrow trephine biopsies than in the lymph node biopsies $(\mathrm{P}=0.0068)$.

\section{Conclusions}

This research identifies the presence of $C D 4+C T L s$ in the $P B$, bone marrow and lymph node biopsies of patients with CLL. The transcription factor profile of CD4+ CTLs was more similar to cytotoxic CD8+ cells than non-cytotoxic CD4+ cells. Although a significantly higher percentage of CD4+CTLs was identified in patients who were CMV seropositive, CMV seronegative patients still retained a measurable population of CD4+ CTLs. The CD4+ CTLs in patients who were CMV seronegative had phenotypic differences when compared with CD4+ CTLs isolated from CMV seropositive patients, which may indicate an alternative, non-anti-viral function. Further research is required to explore whether the alternative function of CD4+ CTLs in CLL is an anti-tumour effect and to investigate the potential to harness them for therapeutic benefit in anti-tumour immunotherapies. 